\title{
Recombinant Fluorescent Rabies Virus Vectors for Tracing Neurons and Synaptic Connections
}

\author{
Nadin Hagendorf and Karl-Klaus Conzelmann
}

\begin{abstract}
Recombinant rabies virus (RV) vectors expressing fluorescent proteins allow staining of neurons from many mammalian species and enable the study of neuron morphology. Because viral spread occurs only between neurons that have synaptic connections, these vectors also permit transsynaptic tracing. A recently established system for restriction of transsynaptic tracing to a single transsynaptic jump, dubbed monosynaptic tracing, uses glycoprotein gene-defective, pseudotyped RV. This allows infection of defined cells and transient complementation with the glycoprotein in situ to support a single step of transsynaptic crossing to presynaptic cells. Here, we introduce protocols describing the production of RV vectors, including the recovery of recombinant RV from complementary DNA (cDNA) and virus pseudotyping in vitro. This allows retrograde staining of neurons projecting to the inoculation site.
\end{abstract}

Rabies virus (RV) is a highly neurotropic, enveloped, negative-strand RNA virus of the Rhabdoviridae family with a very broad host range including virtually all mammals and birds. The major determinant for neurotropism and host range is the viral transmembrane glycoprotein $(G)$, which mediates attachment to target cells, endocytosis, transport of endocytosed vesicles, and virus-vesicle membrane fusion to release the viral nucleocapsid into the cytoplasm of target cells (Finke and Conzelmann 2005; Klingen et al. 2008). Notably, the typical RV retrograde spread from an infected neuron to presynaptic neurons seems to occur exclusively via actual synaptic connections (Ugolini 1995, 2008; Wickersham et al. 2007). This feature is unique among viruses and makes RV a true transsynaptic tracer (Callaway 2008). Transsynaptic transmission requires the $G$ protein, as is evident from recombinant RV lacking the G gene (Street-Alabama-Dufferin [SAD] $\Delta \mathrm{G}$ ) (Mebatsion et al. 1996), which is not able to spread in vitro or in vivo (Etessami et al. 2000). However, the mechanisms behind the specificity of transsynaptic transmission are not yet fully understood. An increased density of high-affinity receptors for the RV G at presynaptic terminals may contribute. So far, p75 neurotrophin receptor (p75NTR) and neuronal cell adhesion molecule (NCAM) have been described as RV attachment and/or receptor proteins, along with gangliosides, phospholipids, and the nicotinic acetylcholine receptor (nAChR; Lafon 2005). Receptor usage of $G$ proteins from different RV strains may differ, and for transsynaptic tracing experiments, the use of $\mathrm{G}$ proteins from virulent (neuroinvasive) RV strains (e.g., challenge virus standard $[\mathrm{CVS}]$ ) is suggested.

Fortunately, nonneuronal cells and cell lines can also be infected in vitro by RV, including hamster kidney cell lines (baby hamster kidney [BHK]) and BSR, a BHK-derived cell line that is used for virus 
stock production. In these nonpolarized cells, budding of free RV particles into the culture supernatant appears to occur all over the cell membrane. When injected into the brain or incubated with brain slice cultures, such supernatant virus predominantly infects neurons, although glia, astrocytes, and oligodendrocytes are also infected by some RV strains.

COMPLEMENTATION, PSEUDOTYPING, AND ENVELOPE SWITCHING

Each of the five genes encoded in the 12-kb RV RNA in the order $3^{\prime}-\mathrm{N}-\mathrm{P}-\mathrm{M}-\mathrm{G}-\mathrm{L}-5^{\prime}$ is essential for unrestricted virus propagation and spread (Fig. 1A). Deletion of the $\mathrm{N}$ (nucleoprotein), $\mathrm{P}$ (phosphoprotein), or L (RNA polymerase) genes leads to failure of RNA synthesis; the lack of G results in the formation of spikeless particles, at least in nonpolarized cells; and in the absence of $M$ (matrix protein), cell-free particles are not produced. Recovery and propagation of such gene-deleted single-round vectors, therefore, requires complementation with the respective gene product in trans, by transient expression or in cell lines expressing the protein (Fig. 1B). G deficiency can be complemented also by analogous envelope proteins of heterologous viruses (e.g., envelope [env] proteins of retroviruses, such as EnvA of avian sarcoma and leucosis virus [ASLV], or HIV-1 Env [gp160]). For effective incorporation of the heterologous spike proteins into the RV envelope, their carboxy-terminal cytoplasmic domains have to be replaced with that of the RV G (e.g., EnvA ${ }^{\text {RGct }}$ ). The resulting pseudotype viruses infect cells according to the receptor specificity of the heterologous env protein (Mebatsion and Conzelmann 1996). The receptor for EnvA (or EnvA ${ }^{\text {RGct }}$ ) is TVA, a small avian protein not present in mammalian cells (Bates et al. 1993). Infection of TVA-expressing

A

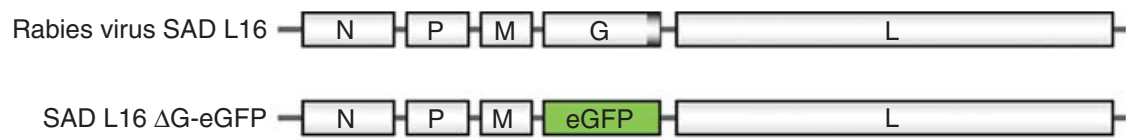

B
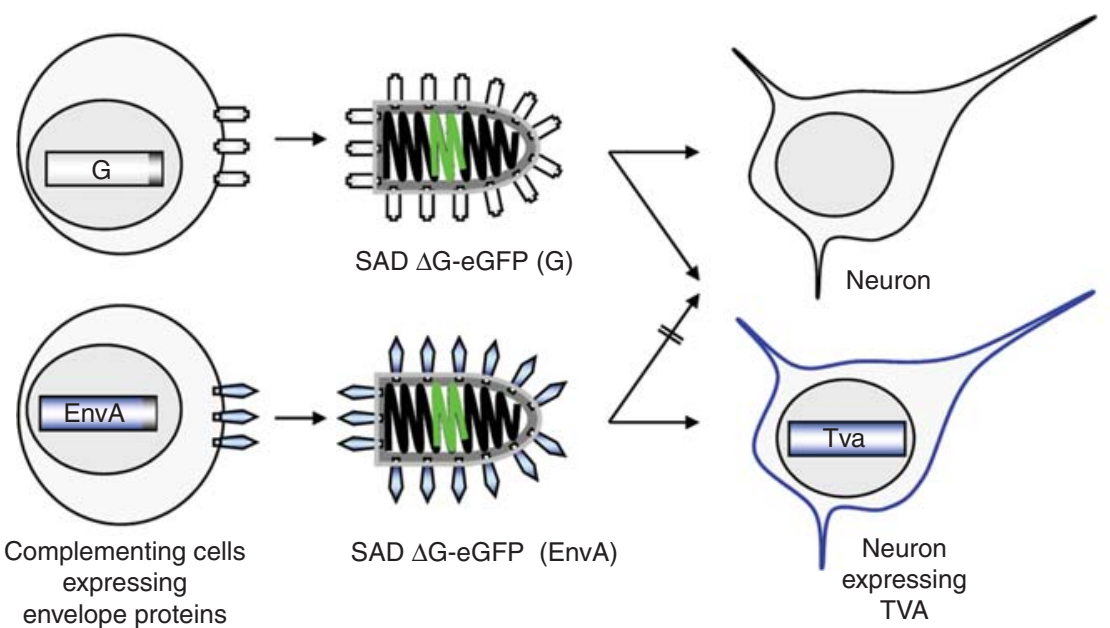

FIGURE 1. (A) Organization of the recombinant rabies virus (RV) RNA genome. The 12-kb RNA of RV (SAD L16) encodes five proteins-nucleoprotein $(N)$, phosphoprotein $(P)$, matrix protein $(M)$, glycoprotein $(G)$, and the RNA polymerase (L). N, P, L, and RNA are associated with the viral ribonucleoprotein (RNP); $M$ forms a layer connecting the RNP with the lipid bilayer membrane; and G is a trimeric type I transmembrane spike protein mediating attachment of the virus to cell receptors and membrane fusion. SAD $\Delta$ G-eGFP lacks a G gene and encodes eGFP (enhanced green fluorescent protein) instead. (B) Pseudotyping of G gene-deficient virus. To yield virus particles able to infect cells, the G deficiency of SAD $\triangle \mathrm{G}$-eGFP must be complemented in cells expressing an analogous glycoprotein (i.e., a protein that binds to a receptor and mediates membrane fusion). For effective incorporation of heterologous proteins, such as the retroviral EnvA, their carboxy-terminal domains should be exchanged with that of G. As EnvA has a highly specific avian receptor (TVA), EnvA-pseudotyped RV infects only mammalian cells engineered to express TVA. 
mammalian neurons by EnvA pseudotype virions is, therefore, highly specific with little background (Federspiel et al. 1994). This combination is, therefore, ideal for monosynaptic tracing experiments such as those pioneered by Callaway and colleagues (Wickersham et al. 2007; Marshel et al. 2010): Neurons expressing TVA and G by standard transgene approaches are selectively infected by RV (EnvA) pseudotype viruses such as SAD $\Delta \mathrm{G}$-eGFP(EnvA). The G-gene deficiency is complemented in the infected cells by the $G$ protein, allowing for a single transsynaptic crossing by the newly formed SAD $\Delta G(G)$ pseudotype viruses. If the TVA-positive target (postsynaptic) cells express a fluorescent protein such as dsRed or mCherry in addition to TVA and G, they can be easily identified by fluorescence microscopy. After infection with SAD $\Delta$ G-eGFP (enhanced green fluorescent protein), they appear yellow in the merge (red and green), whereas the presynaptic cells only stain green from SAD $\Delta$ G-eGFP gene expression (Fig. 2).

\section{GENETIC ENGINEERING OF RV}

The RNA of negative-strand RNA viruses such as RV is not infectious per se, and recovery of the virus from cDNA-derived RNA in cells is a highly inefficient process. The system available involves simultaneous expression of an RNA that corresponds to viral antigenome RNA (cRNA), mostly by T7 RNA polymerase, and three helper proteins $(\mathrm{N}, \mathrm{P}$, and $\mathrm{L})$ to promote assembly of a transcriptionally active nucleocapsid in which the RNA is encapsidated by $\mathrm{N}$ protein and recognized by the coexpressed viral polymerase as a template (Schnell et al. 1994). In contrast to cotranscriptional encapsidation of virus RNA as it occurs during replication of nucleocapsids, such de novo encapsidation of preformed long RNAs by $\mathrm{N}$ is extremely ineffective (Conzelmann 2004). Initiation of an infectious cycle is observed in only one of approximately 100,000 cells expressing cRNA and helper proteins. Because of this low recovery rate, premade amplified virus stocks have to be used in most experiments.

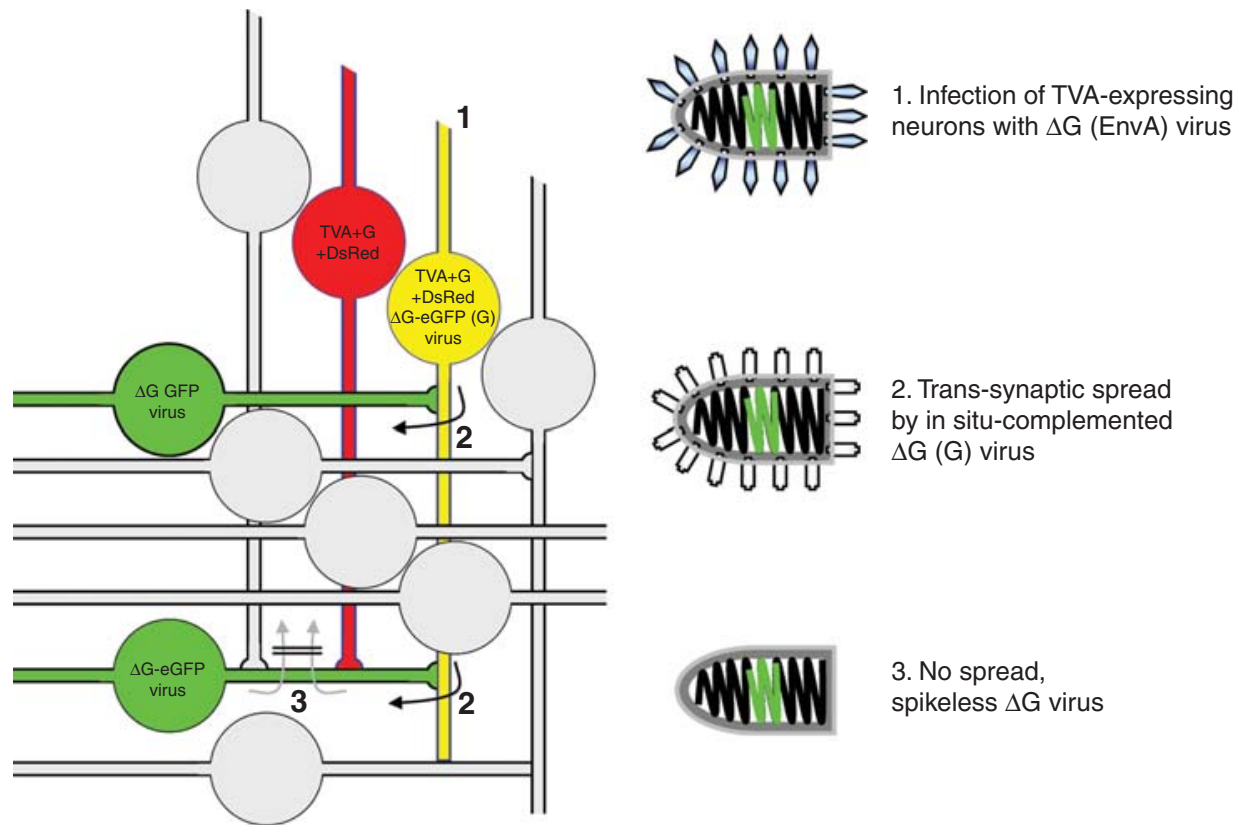

FIGURE 2. Principle of monosynaptic tracing with pseudotyped RV. Neurons are engineered by standard techniques (plasmid transfection, electroporation, gene gun, or transgenics) to express the EnvA receptor TVA, the RV glycoprotein G, and a fluorescent marker (dsRed), thereby allowing identification of potential target cells and infected cells. (1) Successful infection of target cells by EnvA-pseudotyped RV expressing eGFP is observed by yellow (merge) fluorescence; uninfected cells remain red. (2) The G-deficient RV is complemented in situ by the expressed G protein, allowing transsynaptic spread to presynaptic neurons exclusively. (3) The lack of $G$ in infected (green) presynaptic cells prevents spread to secondary order neurons. 
Rabies is a preventable viral illness. All users of RV are advised to read the safety notes and to follow them carefully.

The use and handling of any live virus is hazardous and requires precautions. Because nondeficient RV may cause lethal infection and effective, inexpensive, and completely innocuous vaccines are available, immunization is considered the best solution to prevent any would-be hazard. Genedeleted RVs such as pseudotyped SAD $\Delta \mathrm{G}$ are single-round agents categorized in biosafety level 1 and do not pose a threat per se when handled according to good microbiological practice. The risk of recombination of virus RNA and G mRNA in complementing cells is probably close to 0 because of the tight viral ribonucleoprotein (RNP) and the lack of homologous sequences. Recombination of viral RNA with foreign/cellular RNA (or DNA) has not been observed so far for any of the Mononegavirales (Chare et al. 2003). DNA recombination of viral and complementing G cDNA during rescue experiments, however, has not been excluded. It is, therefore, not advisable to use vaccinia virus for T7 polymerase expression (as used in initial protocols for these studies) because this virus is known to cause high-level plasmid recombination. Also, if the full-length virus is handled in a laboratory, accidental contamination of stocks is possible, and G-deficient viruses obtained from other laboratories may be contaminated as well. In the event of an (a recognized) accident, postexposure prophylaxis is still possible, but prophylactic vaccination is advisable to prevent illness in the event of any such accident. We, therefore, recommend that all personnel working directly with even the G-deleted versions be vaccinated against rabies.

In addition to vaccination, the use of biosafety cabinets, disposable gloves, and protective glasses and the other usual biosafety level 2 personal protective equipment is advised. All materials in contact with the virus (tips, tubes, plates, gloves, gowns, etc.) should be decontaminated by treatment with $70 \%$ isopropanol, and disposable materials should be autoclaved.

\section{EXPERIMENTAL PROTOCOLS}

We present detailed procedures for the recovery of replication-competent RV from cDNA, virus titration, and virus stock preparation, as well as the more demanding recovery of G gene-deleted viruses in Recovery of Replication-Competent and G-Gene-Deleted Rabies Viruses from cDNA (Hagendorf and Conzelmann 2015a). Pseudotyping of G-deficient RV with the neurotropic CVS G protein and ASLV EnvA is described in Pseudotyping of G-Gene-Deficient Rabies Virus (Hagendorf and Conzelmann 2015b).

\section{EXAMPLES OF APPLICATION}

The recombinant G-deleted virus and RV pseudotyped with RV G can be used directly for retrograde staining of all neurons projecting to the inoculation site, both in vitro, as described by Wickersham et al. (2007), and in vivo. By using pseudotype viruses produced in MG139-on cells and, therefore, by carrying the SAD L16 G, which has a less pronounced neurotropism (Finke and Conzelmann 2005), both neurons and glial cells like astrocytes or oligodendrocytes can be infected and identified by fluorescence. The examples in Figure 3 show horizontal cells infected by adding virus to isolated retinas (Fig. 3A) and identified by GFP fluorescence. In the experiment shown in Figure 3B, virus was injected into the spinal cord of a mouse, and explants were analyzed $3 \mathrm{~d}$ after injection. Figure $3 \mathrm{C}$ shows a layer $\mathrm{V}$ neuron after injection into the cortex.

For identification of direct synaptic connections to the cells initially infected by any SAD $\Delta \mathrm{G}$ virus, it is necessary that the missing $\mathrm{G}$ gene is delivered in those cells using standard genetic tools, including cDNA transfection, injection, or delivery by additional viral vectors. The current method of choice for 
N. Hagendorf and K.-K. Conzelmann
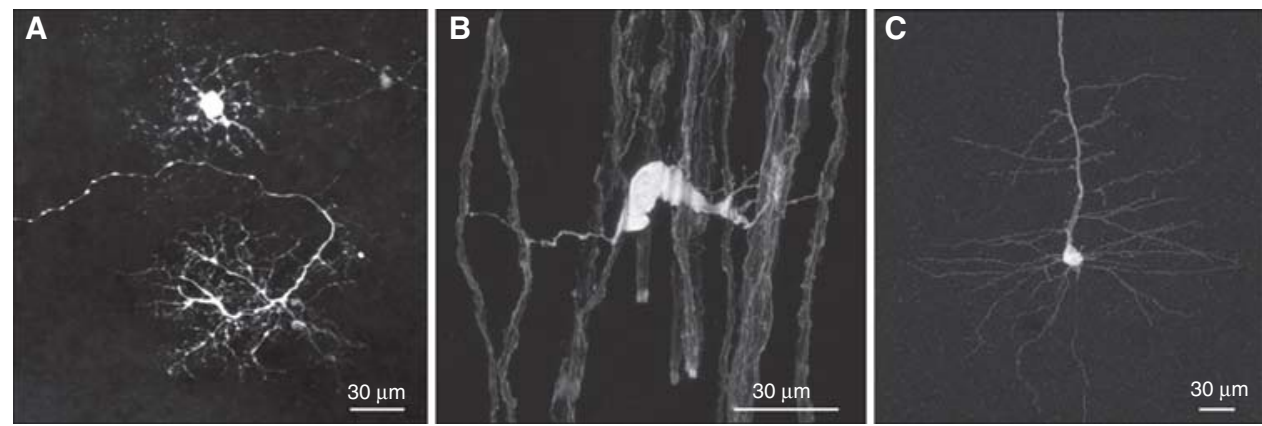

FIGURE 3. Examples of infection of diverse cells types by SAD $\triangle$ G-eGFP $(\mathrm{G})$. (A) Horizontal cells in an explanted mouse retina. (Courtesy of Botond Roska, Friedrich Miescher Institute, Basel.) (B) Oligodendrocyte after injection of SAD $\Delta$ G-eGFP $(G)$ into the spinal cord. (C) Layer $V$ neuron in neocortex. (B,C, Courtesy of Martin Kerschensteiner, Ludwig-Maximilians-University, Munich and Thomas Misgeld, Technical University, Munich.)

monosynaptic (only first-order) connections is the use of SAD $\Delta \mathrm{G}$ (EnvA) pseudotype viruses because this allows unequivocal identification of the starting (postsynaptic) neuron. The latter cell is defined by expression of (1) the receptor TVA to allow specific entry of EnvA-pseudotyped virus; (2) RV CVS$\mathrm{G}$ to allow formation of viruses able to cross the synapse efficiently; and (3) a fluorescent protein to identify the cells. Although few examples are published to date, monosynaptic tracing is being used to study several sensory circuits, including those of the retina, and connections within the spinal cord and cortex.

\section{CONCLUDING REMARKS}

RV vectors allow proficient gene expression in probably all types of neurons and yet cause limited neuronal damage so they do not destroy the connections required for viral spreading to and within the central nervous system. Nevertheless, neurons infected with full-length virus or SAD $\Delta G$ viruses will continuously produce and will accumulate virus RNA and proteins, which ultimately (within a couple of days) will lead to damage. Attempts to engineer vectors with reduced RNA and protein synthesis for a prolonged time window are underway. Future developments are predicted to include the expression of fluorescent proteins targeted to organelles and synaptic structures as well as the expression of functional proteins such as channelrhodopsins and genetically encoded calcium indicators. Although expression of multiple and diverse proteins, including DNA trans-acting factors such as recombinases (e.g., Cre) and transcription factors (e.g., Tet repressors), is straightforward with RV vectors, the use of the respective cis-acting DNA sequences (e.g., lox P and Tet operator) is so far limited to DNA viruses and host cell DNA.

The current poor recovery rate may be sufficient to isolate the desired recombinant virus mutants from transfected cell cultures, but it limits the application of RV for neuronal tracing to work with premade amplified virus stocks. Transfection or injection of neurons with RV cDNA or RNA for standard RV tracing experiments is, therefore, not yet feasible. Similarly, conditional activation of RV cDNA in transgenic animals (e.g., by Cre-mediated recombination) is predicted to be too inefficient for standard tracing experiments.

\section{ACKNOWLEDGMENTS}

Work presented here was funded by the Deutsche Forschungsgemeinschaft through SFB 455 (virus functions and immune modulation) and SFB 870 (assembly and function of neuronal circuits in sensory processing). 
Bates P, Young JA, Varmus HE. 1993. A receptor for subgroup A Rous sarcoma virus is related to the low density lipoprotein receptor. Cell 74: $1043-1051$.

Callaway EM. 2008. Transneuronal circuit tracing with neurotropic viruses. Curr Opin Neurobiol 18: 617-623.

Chare ER, Gould EA, Holmes EC. 2003. Phylogenetic analysis reveals a low rate of homologous recombination in negative-sense RNA viruses. $J$ Gen Virol 84: 2691-2703.

Conzelmann KK. 2004. Reverse genetics of mononegavirales. Curr Top Microbiol Immunol 283: 1-41.

Etessami R, Conzelmann KK, Fadai-Ghotbi B, Natelson B, Tsiang H, Ceccaldi PE. 2000. Spread and pathogenic characteristics of a G-deficient rabies virus recombinant: An in vitro and in vivo study. J Gen Virol 81: 2147-2153.

Federspiel MJ, Bates P, Young JA, Varmus HE, Hughes SH. 1994. A system for tissue-specific gene targeting: Transgenic mice susceptible to subgroup A avian leukosis virus-based retroviral vectors. Proc Natl Acad Sci 91: 11241-11245.

Finke S, Conzelmann KK. 2005. Replication strategies of rabies virus. Virus Res 111: 120-131.

Hagendorf N, Conzelmann K-K. 2015a. Recovery of replication-competent and G-gene-deleted rabies viruses from cDNA. Cold Spring Harb Protoc. doi: 10.1101/pdb.prot089409.

Hagendorf N, Conzelmann K-K. 2015b. Pseudotyping of G-gene-deficient rabies virus. Cold Spring Harb Protoc. doi: 10.1101/pdb.prot089417.
Klingen Y, Conzelmann KK, Finke S. 2008. Double-labeled rabies virus: Live tracking of enveloped virus transport. J Virol 82: 237-245.

Lafon M. 2005. Rabies virus receptors. J Neurovirol 11: 82-87.

Marshel JH, Mori T, Nielsen KJ, Callaway EM. 2010. Targeting single neuronal networks for gene expression and cell labeling in vivo. Neuron 67: 562-574.

Mebatsion T, Conzelmann KK. 1996. Specific infection of CD4+ target cells by recombinant rabies virus pseudotypes carrying the HIV-1 envelope spike protein. Proc Natl Acad Sci 93: 11366-11370.

Mebatsion T, Konig M, Conzelmann KK. 1996. Budding of rabies virus particles in the absence of the spike glycoprotein. Cell 84: 941951.

Schnell MJ, Mebatsion T, Conzelmann KK. 1994. Infectious rabies viruses from cloned cDNA. EMBO J 13: 4195-4203.

Ugolini G. 1995. Specificity of rabies virus as a transneuronal tracer of motor networks: Transfer from hypoglossal motoneurons to connected second-order and higher order central nervous system cell groups. $J$ Comp Neurol 356: 457-480.

Ugolini G. 2008. Use of rabies virus as a transneuronal tracer of neuronal connections: Implications for the understanding of rabies pathogenesis. Dev Biol 131: 493-506.

Wickersham IR, Lyon DC, Barnard RJ, Mori T, Finke S, Conzelmann KK, Young JA, Callaway EM. 2007. Monosynaptic restriction of transsynaptic tracing from single, genetically targeted neurons. Neuron 53: 639-647. 


\section{Recombinant Fluorescent Rabies Virus Vectors for Tracing Neurons and Synaptic Connections}

Nadin Hagendorf and Karl-Klaus Conzelmann

Cold Spring Harb Protoc; doi: 10.1101/pdb.top089391

\begin{tabular}{|c|c|}
\hline $\begin{array}{r}\text { Email Alerting } \\
\text { Service }\end{array}$ & Receive free email alerts when new articles cite this article - click here. \\
\hline $\begin{array}{l}\text { Subject } \\
\text { Categories }\end{array}$ & $\begin{array}{l}\text { Browse articles on similar topics from Cold Spring Harbor Protocols. } \\
\text { Cell Biology, general (1382 articles) } \\
\text { Cell Imaging (525 articles) } \\
\text { Developmental Biology ( } 728 \text { articles) } \\
\text { DNA Delivery/Gene Transfer ( } 344 \text { articles) } \\
\text { Imaging for Neuroscience (342 articles) } \\
\text { In Vivo Imaging (334 articles) } \\
\text { Live Cell Imaging (274 articles) } \\
\text { Neuroscience, general ( } 357 \text { articles) } \\
\text { Viral Methods ( } 113 \text { articles) }\end{array}$ \\
\hline
\end{tabular}

